\title{
LA EDUCACIÓN RURAL Y SUS DESAFÍOS EN EL SIGLO XXI
}

\author{
Sandra Ovares Barquerol \\ Nuria Méndez Garita ${ }^{2}$ \\ Nancy Torres Victoria ${ }^{3}$ \\ Yadira Cerdas Rivera ${ }^{4}$
}

Resumen

El presente artículo centra su interés en los desafios que tiene la educación rural para este siglo XXI; describe el proceso vivido por las poblaciones rurales y como la zona rural, la indígena y los migrantes han sido excluidos. Termina con una serie de propuestas que deben ser incorporadas no sólo en los planes de estudio, sino han de ser consideradas por quienes trabajan en estas regiones.

Palabras claves: retos $y$ desafios, educación, ruralidad.

\section{Abstract}

This article is about the challenges of rural education in the XXI century. It describes the process experienced by rural populations in Costa Rica and the exclusion suffered by indigenous and immigrants. At the end, the author offers different proposals that could be incorporated not only in educational curricula but also as a guide for professionals who work in these regions.

Keywords: challenge, education, rural context.

1 Máster en Administración Eủisativa. con especialidad en Curriculum, Enseñanza de las Ciencias y la Biologia. Experiencia en la formaciọn de maestros para comunidades indigenas. Actualmente es académica e investigadora de la División de Eduizción Rural. CIDE, UNA.

2 Mảster en Literatura Latincanzericana. con especialidad en la Enseñanza del Español. Experiencia en formación de maestros para las comuniujaues rurales. Actualmente es la directora de la Revista EDUCARE y académica investigadora de la Division de Educación Rural. CIDE, UNA.

3 Licenciada en Historia. investigadora, consultora, con experiencia en Derechos Humanos. Académica de la División de Educación Rural.

4 Máster en Docencia. Con fonnación en I y II ciclos; investigadora y académica de la División de Educación Rural, donde actualmente. oiupa el cargo de Directora.

Recibido: 10 de marzo-200- $\cdot$ Aprobado: 30 de abril-2007 


\section{La educación rural y sus desafíos en el siglo XXI}

El modelo de acompañamiento social participativo dista mucho de ser una invasión cultural.

Toda invasión sugiere, obviamente, un sujeto que invade su espacio histórico-cultural, que le da su visión de mundo. en el espacio desde donde parte, para penetrar otro espacio histórico-cultural, imponiendo a los individuos de éste, su sistema de valores. El primero actía, los segundos tienen la ilusión de que actúan, en la actuación del primero, este dice la palabra; los segundos prohibidos de decir la suya. escuchan la palabra del primero.

El invasor piensa, en la mejor de las hipótesis sobre los segundos, amás como ellos, estos son "pensados" por aquellos. El invasor prescribe, los invadidos son pasivos frente a su prescripción.

Pablo Freire

A ntes que nada, es importante aclarar que el derecho a la educación debe ser considerado un derecho inalienable de todos los seres humanos. La educación como derecho humano y bien público permite a las personas ejercer sus otros derechos. Por esta razón, nadie puede quedar excluido de ella. Como lo plantea la UNESCO (2007): "El derecho a la educación se ejerce en la medida que las personas, más allá de tener acceso a la escuela, puedan desarrollarse plenamente y continuar aprendiendo. Esto significa que la educación ha de ser de calidad para todos y a lo largo de la vida"5.

Así entiende la educación y el derecho a la educación, la División de Educación Rural; se conciben en el ejercicio diario del quehacer educativo que inició con la creación de la universidad necesaria: la Universidad Nacional; revolucionaria idea de Benjamín Núñez y otros pensadores pedagogos costarricenses en 1973. "A partir de allí, se aboga porque la tarea del proceso de construcción de conocimientos esté orientada hacia la interpretación de la realidad y en constante reorganización a la par de las aspiraciones de sus miembros" (Cerdas, 2006, p. 159).

Mas, ¿la construcción de conocimientos orientada hacia la interpretación de cuál realidad? ¿Y en constante reorganización, con la participación

5 Documento de discusión sobre politicas cducativas en cl marco de la II Reunión Intergubemamental del Proyecto Regional de Educación para América Latina y el Caribe (EPT/PRELAC), que fue creado entre el 29 y 30 de marzo de 2007; Buenos Aircs, Argentina. (U) UNESCO, Oficina Regional de Educación para América Latina y el Caribe (OREALCIUNESCO, Santiago). 
y aspiraciones de los miembros de cuáles comunidades? ¿Y una educación para qué? Estas y otras interrogantes están asociadas con la discusión sobre los desafios que enfrenta la educación en general en la actualidad, y la educación rural en particular.

Lo rural se ha entendido como sinónimo de atraso. La ignorancia y la pobreza han sido asociadas, tradicionalmente, con lo rural, y fueron entendidas por los responsables de diseñar las políticas en general -pero sobre todo, las políticas educativas- como el resultado de su interés por mantener intactas las tradiciones, por lo que la educación modemizante urbanizante fue imaginada como la vía necesaria para alcanzar el progreso y, fundamentalmente, el desarrollo económico.

Sin embargo, lo rural se entiende hoy más ampliamente. El concepto ha trascendido incluso más allá de lo que se concebía en el sector agropecuario. Pomareda afirma que "el medio rural es el conjunto de formaciones socioespaciales locales y regionales reconocibles por la baja densidad poblacional que presentan el predominio de la agricultura y el bosque, con presencia de una importante diversidad de recursos naturales, todavía poco aprovechados pero potencialmente aprovechables para el mejoramiento de la calidad de vida de la población" (1993, p. 46).

El medio rural costarricense, por lo general, presenta una baja densidad poblacional y posee menos bienes y servicios que las ciudades, o los posee en condiciones de calidad deficitaria. Los habitantes de las zonas rurales tienden a valorar, mantener y respetar las relaciones familiares y su contexto natural. Se vive en una cultura en la cual sobresalen manifestaciones cotidianas de solidaridad, austeridad y sencillez de costumbres, lo que permite todavía percibir, en la vida de algunas poblaciones rurales, un ritmo sosegado.

Es también característica de las zonas rurales costarricenses, la poca organización comunal de sus pobladores para enfrentar los problemas de sus comunidades y el lento conocimiento de los mecanismos que pueden utilizarse para canalizar sus inquietudes. Sin embargo, si se observa con cuidado, la organización comunitaria existente tiene su base social en la iniciativa y participación de las mujeres creativas que vienen abriendo nuevos espacios en la búsqueda de fuentes de trabajo, apoyando la llegada y el establecimiento de nuevas altemativas educativas en la educación secundaria por ejemplo, fundando comités, ONGs y organizaciones en las cuales se agremian para luchar por sus proyectos y demandas.

La falta de fuentes de trabajo, de oportunidades educativas, culturales, comerciales y la poca variedad, generan una alta migración a las ciudades. 
El mal estado de los caminos y la participación de intermediarios impiden obtener, también, beneficios económicos de la siembra de productos.

Por otro lado, las poblaciones rurales no son homogéneas, como bien lo describe Céspedes (2005); ciertamente, las hay que siguen dependiendo del agro, en esquemas de pequeña propiedad y de autoabastecimiento y hay también comunidades rurales en zonas bananeras, piñeras y otras en las cuales la mayoría de sus habitantes son empleados de grandes compañías transnacionales. De igual forma, están los habitantes de comunidades pesqueras, localizadas en la costa pacífica y en la atlántica; comunidades indígenas, las cuales, además de las características anteriores, tienen una dinámica y forma propias para enfrentar y entender la vida; comunidades con importante presencia de grupos de inmigrantes, comunidades rurales dispersas, comunidades costeras, rurales y turísticas, comunidades dedicadas al turismo rural, al turismo ecológico, entre muchas otras.

Como características de las zonas rurales se han señalado, por tradición, un paisaje cargado de naturaleza, el trabajo de campo (siembra, cosecha, cuido y cría de animales, producción agrícola), la escasez de servicios básicos, la lejanía entre las viviendas y los centros poblacionales, las grandes distancias para trasladarse prácticamente a cualquier sitio, la falta de lugares o medios de recreación y esparcimiento, el papel de la escuela y la iglesia como centros sociales, el bajo nivel de escolaridad de sus pobladores y la pobreza.

No obstante, los espacios rurales cambian y se transforman de una manera acelerada, por lo que algunas de las características anteriores, ya no corresponden con el nuevo escenario. La percepción que antaño imperaba sobre lo que el mundo rural era y demandaba es hoy, en la mayoría de regiones, un supuesto histórico superado; las comunidades rurales de la actualidad reclaman otras necesidades y se adaptan a los cambios que los grupos humanos del nuevo milenio experimentan.

La realidad rural de hoy es compleja y cambiante. Los cambios en la explotación de los recursos naturales han transformado los patrones de vida de las familias del medio rural. A esto, se deben sumar los cambios culturales, migratorios y demográficos de las últimas décadas, que han vuelto más compleja la vida rural.

El campo es cada vez menos agrícola y más turístico; la participación de la mujer en las actividades productivas empieza a influir fuertemente en la transformación de la vida familiar campesina; y se vive un importante proceso de empobrecimiento que obliga a muchas familias a sacar a las niñas y 
los niños de la escuela para incorporarlos al trabajo informal. Esta situación refuerza el pensamiento que, una vez terminado el segundo ciclo, es decir el sexto grado, se ha culminado con el proceso educativo de las niñas y los niños (Céspedes, 2005, p. 30).

En el marco de la situación actual, más que nunca, se espera que la educación rural y la escuela rural, como centros del quehacer educativo, desempeñen el rol determinante que la sociedad históricamente les ha asignado desde sus inicios. Es decir, enseñar para la vida, entregar una educación pertinente, transitar por experiencias que permitan compromiso social y desarrollo de las relaciones escuela-comunidad.

Desde 1918, al terminar la administración Tinoco, Roberto Brenes Mesén escribió, como introducción a los programas diseñados específicamente para las escuelas rurales de Costa Rica:

"Para la ejecución de los presentes programas de Educación Primaria con destino a las escuelas rurales se han contemplado los más graves problemas nacionales desde diversos puntos de vista y contienen, por lo tanto, implícitamente, las soluciones más eficaces y mejores. La escuela rural está concebida aquí como un centro de creación de riqueza agrícola e industrial, al mismo tiempo que como un medio de educación positivista de virtudes activas" (Ramírez, 1996, p. 263).

Este documento se constituye en la prueba de que la escuela rural ha sido y es uno de los pilares sobre los cuales se ha construido la educación costarricense desde los inicios de la historia de la educación en este país.

Las escuelas rurales, como tales, existían ya desde 1881, pero sus momentos de auge se dan en 1886 y en 1906, cuando se invierte en una mayor infraestructura en esas zonas. En la década de los años cincuenta, la Misión de la UNESCO desarrolló un proyecto de educación rural en la zona de los Frailes y San Isidro de El General, que luego se amplía a Guápiles y San Carlos (Ramírez, 1996, p. 269).

En el marco de este proyecto, se implementó el programa de Nutrición y Huertas Escolares, que es uno de los proyectos que mayor impacto ha tenido en las zonas rurales. El mejor resultado se obtuvo en la coordinación que se pudo lograr entre los ministerios de Salud, Educación y Agricultura y con los organismos internacionales de la UNICEF y la FAO.

El proyecto impulsó el cultivo de productos agrícolas en las huertas escolares, para lo cual se hizo necesaria la capacitación del personal docente, con 
el apoyo del Ministerio de Agricultura y la FAO; la UNICEF donó las herramientas, los abonos y las semillas. Así, los productos se consumían en los comedores escolares, con la participación del Ministerio de Salud, organizando los aspectos de salubridad y capacitando en manejo de alimentos (Ramírez, 1996, p. 270). En las décadas de los 70 y los 80, la proporción de escuelas rurales creció en forma importante, y en los años 90 la tendencia se mantenía.

Sin embargo, es importante destacar que a partir de 1984, se presentó un importante faltante de docentes para atender la demanda educativa en las zonas rurales. Para dar respuesta a esta situación, el Ministerio de Educación propone un convenio a las tres universidades públicas: Universidad de Costa Rica, Universidad Estatal a Distancia y Universidad Nacional de Costa Rica, con el fin de que se diseñara y se aplicara un plan curricular de formación de docentes rurales que combinaba teoría y práctica, mediante la metodología de investigación-acción. Visualizaba el aula como un laboratorio en el cual se aplican nuevas e innovadoras estrategias metodológicas que luego se analizan en las sesiones presenciales, docentes y estudiantes universitarios en conjunto, a la luz de las teorías pedagógicas que partían del postulado de la participación y fortalecimiento de lo local.

Según datos del Departamento de Estadísticas del MEP, para el 2006 existían en Costa Rica 4.027 escuelas de primaria, de las cuales 3.250 son escuelas rurales, incluyendo en esta cifra las escuelas unidocentes, que son 2.981; es decir, el $97 \%$ de las escuelas son rurales (Construcción propia a partir de estadísticas del MEP). Cabe aclarar que, aunque son una mayoría, sólo atienden el $8 \%$ de la matrícula total nacional, lo que equivale a unas 42.000 personas aproximadamente.

Si bien la educación primaria tanto en las áreas urbanas como en las rurales es un tema que podría darse por superado en el país, no se puede afirmar lo mismo de la secundaria. La situación es aún más crítica cuando hablamos de las áreas rurales. En este campo, se puede ubicar uno de los desafíos más importantes de la educación rural en Costa Rica: la cobertura educativa secundaria en las áreas rurales es la gran tarea pendiente.

Según el I Informe del Estado de la Educación, para enfrentar los desafios actuales del desarrollo humano tanto desde el punto de vista productivo como del político y el social, se toma cada vez más relevante el debate sobre el aporte de la educación y el conocimiento al desarrollo y fortalecimiento de las capacidades humanas. El sistema educativo costarricense no ha logrado garantizar una cobertura universal de la educación general básica, los niveles observados en el primer ciclo caen a medida que aumenta el nivel educativo. 
En general, es en el tránsito de la primaria a la secundaria y entre ciclos donde se registran los mayores niveles de no asistencia, repitencia y abandono. "A mediados de la década de los noventa empezó a realizarse un esfuerzo importante por recuperar los niveles de inversión en educación, cobertura e infraestructura escolar y se obtuvo una mejora en todos estos indicadores. Así en el 2002 se logró un $60 \%$ de cobertura en secundaria, con lo que se recobró el nivel de 1980" (2005, p. 68).

Como bien lo recomienda el mismo informe, se hace prioritario hoy actuar en los ámbitos donde las diferencias y discriminaciones son más evidentes. En las áreas rurales, se ha instalado una enorme capacidad educativa. Esto quiere decir que se cuenta con instalaciones educativas, para su desarrollo administrativo y organizativo, y se cuenta con la presencia de un docente nombrado por el Estado, quien atiende a la población estudiantil.

Si bien la cobertura en la primaria es un tema al que el país le ha apostado en serio, se devela otra, no menos trascendente: el tema de la cobertura, calidad, promoción, retención y permanencia en la secundaria. Como se dijo, se convierte en uno de los desafíos más agudos de la educación costarricense, pues no se logra responder aún a las necesidades educativas de la población adolescente en las áreas urbanas y en las áreas rurales esta necesidad es todo un reto, que el país está en mora de abordar.

La educación secundaria en las áreas rurales debe colocarse como una de las prioridades por atender en forma inmediata. Aunque hoy se han implementado nuevas ofertas y modalidades para atender la educación secundaria en las áreas rurales, como la Telesecundaria, bachillerato a distancia, educación abierta, queda aún un enorme vacío en este tema. La oferta educativa en las zonas rurales debe responder con currículos pertinentes, con salidas colaterales técnicas ligadas a formas productivas y que preparen de forma más inmediata y a más corto plazo para el trabajo, de lo contrario, la educación seguirá siendo en las zonas rurales el talón de Aquiles del tema educativo.

Por otro lado, está el tema de la calidad educativa. Es muy documentada la brecha existente en la calidad, la cobertura, la eficiencia y la eficacia de la educación urbana y la educación rural. El IX Informe del Estado de la Nación aclara que las diferencias entre lo urbano y lo rural se mantienen y en algunos grupos etarios incluso se incrementan:

“El año 2002, en el grupo etario de 15 a 17 años, que se esperaba tuvieran niveles de escolaridad cercanos al final del tercer ciclo, o algún nivel de ciclo diversificado, se observó que, tres de cada diez jóvenes de zonas urbanas, tenían algún grado de primaria, proporción que en 
las zonas rurales asciende a solo uno de cada dos jóvenes. Para quienes provenían del área urbana, casi un $20 \%$ se había asegurado algún nivel de escolaridad en educación diversificada, en contraste con solo cerca de un $10 \%$ en las áreas rurales" (2002, p. 38).

El anterior análisis presenta un gran reto: se debe alcanzar y consolidar la cobertura universal de la educación básica para las presentes y futuras generaciones, los rezagos educativos inciden y determinan las posibilidades de movilidad social y el bienestar presente y futuro de las personas. Los datos del IX Informe del Estado de la Nación demuestran que existe un ingreso promedio mayor en la zona urbana que en la rural y directamente relacionado con la variable que mide el nivel de escolaridad. De igual manera, se nota un vínculo entre el tipo de trabajo realizado y el nivel de escolaridad. Entre los jóvenes que no alcanzaron algún grado de educación general básica, la mayoría (más del 95\%) se ubicaba en las categorías ocupacionales de más baja calificación.

Visto así, se hace urgente que se tomen diferentes acciones para propiciar mayores alternativas educativas en secundaria para las zonas rurales. Pero no cualquier iniciativa, sino una educación primaria y secundaria pertinente, atinente, propia, que responda a intereses y necesidades, que respete contextos, historias, acervo cultural y tradiciones, la multiculturalidad, los liderazgos, sabidurías y saberes, formas de hacer y aprender locales.

Los habitantes de las áreas rurales de Costa Rica tienen menos oportunidades de ejercer su derecho de una educación gratuita y obligatoria, que debe ser garantizado y ofrecido por el Estado. Para dar respuesta al conjunto de necesidades educativas de las áreas rurales, se hace necesario, además de la decisión política por parte del Estado de intervenir en estas áreas, el diseño de políticas de intervención, el establecimiento de planes y programas y de múltiples instrumentos y herramientas didácticas, metodológicas y pedagógicas que permitan hacer realidad el ejercicio del derecho a la educación de las personas que habitan en las zonas rurales.

En este sentido, la División de Educación Rural (DER), en el Centro de Investigación y Docencia en Educación (CIDE), juega un rol importante, ya que es la única institución en Centroamérica que tiene como objeto de estudio y misión la formación de docentes especializados en la educación rural.

Desde el enfoque de la pedagogía rural de la DER, el o la docente rural se concibe como un gestor comunitario, un dinamizador de procesos sociales en las comunidades rurales. Los y las docentes de las escuelas rurales deben ser más que maestros; han de trascender el quehacer del aula y visualizar la 
comunidad y sus necesidades como su campo de acción, ejercer procesos de liderazgo, diseñar proyectos que nazcan y que respondan a las necesidades de la comunidad, que promueva y que fortalezca procesos de educación no formal, que sea capaz de investigar y de hacer una lectura constante de la realidad de su aula y de su entomo, con el fin de que la entrega de la docencia sea pertinente y de calidad.

El enfoque que se ha venido construyendo en la DER, sobre la educación rural, es el de una educación rural diseñada desde la identificación de las necesidades educativas de las comunidades rurales, mediante procesos participativos. Se elimina el mito urbanocéntrico que orienta las políticas educativas en el campo de la educación, que parte del supuesto (equivocado...) de que cualquier educación debe o puede servir para modernizar a los "atrasados pueblos indigenas y campesinos de las zonas rurales".

Este modelo sólo ha traído desarraigo, migración hacia las ciudades, consumismo, homogeneización, pérdida o debilitamiento de la riqueza y de la diversidad cultural existente en el territorio nacional, venta de terreno rural a bajo precio, empobrecimiento y miseria a los habitantes de las zonas rurales $y$, por ende, de la sociedad en general.

En las políticas educativas, se destaca la necesidad de atender la educación rural de manera urgente. Sin embargo, los planes se quedan sin contenido presupuestario. Se requiere concentrar esfuerzos y voluntades de instituciones que demanden y exijan que se pase de las declaraciones programáticas y discursos a la ejecución con participación en las comunidades rurales de lo planteado.

Por otro lado, y atendiendo a los cambios en un mundo globalizado, antes de abogar por introducir las Tecnologías de la Información y Comunicación (TICs) en las comunidades, es importante discutir cuál podría ser el papel que van a desempeñar estas y cómo darán respuesta a las múltiples necesidades de la educación rural. Esta es una tarea para educadores, con participación amplia de las comunidades educativas de las zonas rurales.

Las propuestas curriculares integradoras son un desafio para la educación rural, pues toman en consideración las dimensiones globales de la sociedad. Una sociedad en la que los medios de comunicación y las redes informatizadas son unos de los principales motores que entrelazan todas sus dimensiones: vida económica, cultural, productiva, política y otras. El objetivo fundamental de la integración curricular es preparar al o la estudiante para ser un(a) ciudadano(a) activo(a) y crítico(a), que le permita tomar decisiones, para asumir los retos que le presenta el mundo actual. 
Para ir finalizando, cabe decir que el desafio más importante de la educación rural está en que seamos capaces, maestros, campesinos, antropólogos, sociólogos, madres, padres, estudiantes, líderes comunitarios, mujeres, niñas, niños, adolescentes, trabajadores de la salud, es decir, todas las personas que tenemos algún interés y relación con lo rural, de interpretar y hacer lectura de los saberes instalados desde siempre en la ruralidad costarricense, para construir juntos la educación, el currículo, la metodología, los insumos didácticos, los textos, los espacios, los proyectos que están necesitando quienes habitan en las áreas rurales de Costa Rica. No sólo tiene derecho a habitar allí, sino que quiere seguir allí, y el Estado debe garantizar -tal como se ha comprometido a hacerlo-su derecho a recibir una educación de calidad, gratuita y obligatoria.

Como universidad, uno de los desafios que afrontamos hoy los y las docentes de la División de Educación Rural, es la revisión permanente de los planes y mallas curriculares de formación, a partir de la investigación de la realidad del país, procurando la participación de las comunidades, desde el riguroso análisis de las prácticas pedagógicas posibles en los contextos rurales. Además, somos conscientes de la importancia de que los docentes participen en procesos de formación continua. La actualización permanente nace a raíz de esa lectura que cada maestro y maestra deben hacer de los cambios que se dan en la sociedad. 


\section{REFERENCIAS}

Aguilar, M. y Monge, M. (2000). Hacia una pedagogía rural. FUNA.

Aguilar, María Esther et al. (2000). Educación rural: un acercamiento. FUNA.

Cerdas Rivera, Y. (2006). Educación y Desarrollo: Un homenaje al doctor Jan Ooijens. Versión en CD. CIDE. DER. UNA.

Céspedes Ruiz, E. y Torres, V. N. (2005). "Acerca de la educación rural en Costa Rica". Artículo publicado en la revista Aportes $N^{o} l$ de marzo que publica regularmente IDESPO. UNA. Costa Rica.

Freire, P. (1970). Pedagogía del oprimido. Río de Janeiro: Paz e Terra.

Freire, P. (2002). Pedagogia de la esperanza. México: Siglo XXI.

Pomareda, C. (1993). La agricultura en el desarrollo económico de Centroamérica en los 90. Editorial IICA.

Proyecto Estado de la Nación. (2002). IX Informe Estado de la Nación en Desarro-

llo Humano Sostenible. Costa Rica: Proyecto Estado de la Nación.

Proyecto Estado de la Nación (2005). I Informe del Estado de la Educación. San José.

Ramírez Rivera, M. (1996). Educación en las áreas rurales de Costa Rica. Proyecto UNESCO Fortalecimiento de la Educación en las Áreas Rurales del Istmo Centroamericano. Heredia, Costa Rica. UNESCO.

Revista Educare. Monotemático: educación y ruralidad. N 3-2002. EUNA.

Solano, J. et al. (2003). El docente rural en Costa Rica: radiografia de una profesión. EUNA.

Torres, J. (1997). "Política educativa, multiculturalismo y prácticas culturales democráticas en las salas de aula". Revista Brasileira de Educação. (São Paulo - Brasil), nº 4 (Janeiro-Abril).

Torres, Jurjo. (1998). Globalización e interdisciplinariedad: el curriculum integrado. Madrid: Ediciones Morata.

Van der Bijl, B. (2000). La escuela y la comunidad rural. FUNA. 